\title{
Effect of Rapid Thermal Annealing on the Photoluminescence from Si Nanocrystal Decorated Si Nanowires Array Grown by a Metal Assisted Chemical Etching Method
}

\author{
Ramesh Ghosh ${ }^{1}$, P. K. Giri ${ }^{1,2 *}$ \\ ${ }^{1}$ Department of Physics, Indian Institute of Technology Guwahati, Guwahati -781039, India \\ ${ }^{2}$ Centre for Nanotechnology, Indian Institute of Technology Guwahati, Guwahati -781039, \\ India
}

\begin{abstract}
In this report, the micron-long Si nanowires (NWs) array is grown by a metal assisted chemical etching (MACE) process using $\mathrm{Ag}$ as the noble metal catalyst in $\mathrm{HF} / \mathrm{H}_{2} \mathrm{O}_{2}$ solution. These Si NWs are decorated with arbitrary shaped ultrasmall Si nanocrystals (NCs) due to the side wall etching of the Si NWs. The MACE grown samples exhibit strong PL emission in the visible region and quantum confinement (QC) effect of photon in the Si NCs is believed to be the dominant mechanism behind PL. We have investigated the influence of rapid thermal annealing with different annealing environments on the optical properties (such as PL, Raman and reflectivity) of the Si NCs decorated Si NWs samples to gain better insight into the QC effect. The effect of laser excitation power on the PL properties of the as-grown Si NCs/NWs sample are also inspected in this report for further confirmation of QC effect behind the PL emission profile.
\end{abstract}

Keywords: Si nanocrystal, Rapid thermal annealing, Photoluminescence, Quantum confinement, Oxygen vacancy, Compressive strain.

\section{INTRODUCTION}

\footnotetext{
* Corresponding author, email: giri@iitg.ernet.in
} 
Among the low-dimensional semiconductors, Si nanowires (NWs) have always been considered to be very important because $\mathrm{Si}$ is the most abundant and widely used semiconductor, with a large industrial infrastructure for the low-cost and high-yield $\operatorname{processing}^{1-4}$. Si NWs grown by metal assisted chemical etching (MACE) process are usually decorated with arbitrary shaped Si nanocrystals (NCs) that exhibit significantly different optical characteristics from the bulk Si counterpart, including Raman spectra, visible to near infra-red (NIR) photoluminescence (PL) and reflectance and these properties are exploited for variety of applications, such as LED, sensors and high efficiency photovoltaic cells ${ }^{1-5}$. Based on quantum confinement (QC) effect, these MACE grown Si NWs decorated with arbitrary shaped and nano-sized Si NCs can emit visible PL depending on the size of the Si $\mathrm{NCs}^{5-7}$. These Si NCs decorated Si NWs are covered with a very thin layer of $\mathrm{SiO}_{\mathrm{x}}(1<\mathrm{x}<2)$ and the defects in the $\mathrm{Si} / \mathrm{SiO}_{\mathrm{x}}$ interface can also produce the visible PL emission ${ }^{8,}{ }^{9}$. It becomes necessary to adopt some techniques to remove or improve upon such defects. Rapid thermal annealing (RTA) is often used to improve the structural properties of the material ${ }^{10}$. However, effect of RTA on the MACE grown Si NWs in its optical properties are not studied in the literature.

Here, we have grown Si NWs decorated with Si NCs by MCAE and performed RTA to improve the structural and optical properties of as-synthesized Si NWs. We have studied the influence of RTA treatment with different annealing environment (vacuum, nitrogen and oxygen) on the PL properties of Si NCs decorated Si NWs. We have performed the reflectivity and Raman analysis on the Si NCs/NWs before and after RTA treatment. The effect of laser heating on PL spectra was also studied for confirmation of QC effect.

\section{EXPERIMANTAL DETAILS}

Si NWs are grown from boron-doped p-type $\mathrm{Si}(100)$ wafers with resistivity $0.01 \Omega$ $\mathrm{cm}$. The Si wafers are initially cleaned by rinsing in acetone followed by ethanol for $5 \mathrm{~min}$ in 
each step. The wafers are cleaned in piranha solution (a 5:1 mixture of sulfuric acid $\left(\mathrm{H}_{2} \mathrm{SO}_{4}\right)$ and hydrogen peroxide $\left.\left(\mathrm{H}_{2} \mathrm{O}_{2}\right)\right)$ for $10 \mathrm{~min}$ to remove the metallic and alkaline contamination as well as for the reduction of organic residues on the wafer surface. Native oxide layer $\left(\mathrm{SiO}_{2}\right)$ is removed by immersing the wafers in $10 \%$ hydrofluoric acid (HF) for a few minutes. The samples are rinsed in de-ionized (DI) water after each step. Finally the cleaned wafers are dried by blowing Ar gas. For etching of the wafers, we have used a two-step metal assisted chemical etching (MACE) process. At first, a thin layer of silver (Ag) nanoparticles is formed on the $\mathrm{Si}$ substrate by dipping the substrate in a solution containing $0.015 \mathrm{M}$ $\mathrm{AgNO}_{3}$ and 5.55 M HF for a few seconds and then the substrate is immersed in solution containing $1.422 \mathrm{M} \mathrm{H}_{2} \mathrm{O}_{2}$ and 4.6 M HF for $20 \mathrm{~min}$ (sample $\mathrm{S} 1$ ). The sample $\mathrm{S} 1$ is cut into pieces and rapid thermal annealing (RTA) (Mila 3000P, ULVAC) is performed for $60 \mathrm{sec}$ in $800{ }^{\circ} \mathrm{C}$ for three different annealing environments. Details of the sample codes and RTA parameters of the Si NC decorated Si NWs are shown in Table I. The morphologies of the Si NWs are characterized using a field emission scanning electron microscope (FESEM) (Sigma, Zeiss) and a transmission electron microscope (TEM) (JEOL-JEM 2010) operated at $200 \mathrm{kV}$. For structural characterizations, we have used high resolution TEM (HRTEM) equipped with an energy-dispersive X-ray spectrometer (EDX) and a Gatan Digital Micrograph system. The diffuse reflectivity of the samples is carried out using ellipsometry (GES5-E, SEMILAB Sopra). The steady state photoluminescence (PL) spectrum is recorded using a $405 \mathrm{~nm}$ DPSS (Diode-Pumped Solid State) laser excitation with the help of a spectrometer (Thermo Spectronic AMINCO-Bowman Series 2 spectrometer, FA-357) equipped with a PMT detector. Raman measurements are carried out with a $633 \mathrm{~nm} \mathrm{Ar}^{+}$laser excitation using a micro-Raman spectrometer (LabRAM HR-800, Jobin Yvon).

\section{RESULTS AND DISCUSSION}

\subsection{Growth and Morphology}


The growth studies of $\mathrm{Si}$ NCs decorated $\mathrm{Si}$ NWs or the porous Si NWs using electroless two-step MACE are reported by several groups ${ }^{5,11}$. The formation mechanism of the Si NCs on the surface of the Si NWs strongly depends upon the porous structures of the NWs. The shape and size of the Si NCs depend on the size of the pore and intermediate distance between the pores ${ }^{5}$. Figure 1 (a) and (b) show the FESEM top and cross-sectional micrographs acquired from as-grown samples S1. Fig. 1(c) shows the corresponding TEM image of individual Si NW. It is clear that the Si NWs surface is rough due to the presence of the Si NCs originating due to the side-wall etching. Figure 1(d) shows the TEM image of single Si NW acquired from sample S1_O2. A thin layer $(\sim 13 \mathrm{~nm})$ of $\mathrm{SiO}_{2}$ is formed on the surface of the Si NWs due to oxidation of Si. Figure 1(e) shows the selected area diffraction (SAED) pattern of the sample S1 while Fig. 1(f) shows the corresponding HRTEM image of the surface of the Si NWs. The typical dimension of a single Si NC is marked by white dotted line. It is clear from Fig. 1(e) and 1(f) that the Si NCs are highly crystalline and the calculation of d-spacing in Fig. 1(e) shows a reduced lattice constant (2.94 ̊), implying a compressive strain in the lattice. This may be related to the native oxide layer grown on the $\mathrm{Si}$ NWs/NCs surface. The SAED pattern in Fig. 1(e) confirms that several crystal planes are present in the Si NC/NWs lattice structure.

\subsection{Optical reflectivity}

The absorption coefficient of the Si NW/ NCs is assessed by measuring the diffused reflectivity under oblique incidence of the samples. Figure 2 shows a comparison of the optical reflectivity as a function of photon energy for the bulk Si wafer and Si NWs decorated with $\mathrm{Si}$ NCs before and after RTA treatment. The samples show significantly lower reflectivity at all wavelengths as compared to the bulk Si wafer. Due to multiple reflections on the inner surface of the Si NWs and a broad range of size distribution of the Si NCs, the absorption is significantly high in the case of $\mathrm{Si} \mathrm{NWs} / \mathrm{Si}$ NCs over the entire range of 
wavelength $^{1,5}$. Note that the MACE grown Si NWs may have residual Ag nanoparticles (NPs) lying at the bottom of the NWs and partly at the surface of the NWs/NCs. These Ag NPs may cause higher absorption in the visible range due to the surface plasmon resonance $(\mathrm{SPR})^{12}$ effect. However, since the NWs were etched in $\mathrm{HNO}_{3}$ solution after growth, their contribution may not be significant, though it is not negligible. Due to the high absorption over a broad range of wavelength, such nanostructures are highly beneficial for photovoltaic application requiring antireflective properties over the entire visible-NIR range ${ }^{1}$. The RTA treated sample S1_O2 shows the highest absorption over the entire visible range.

\subsection{Photoluminescence}

Photoluminescence spectroscopy is a contactless, nondestructive method of probing the electronic structure of materials and detailed PL study on nanostructured Si can provide useful information about the purity, amorphicity, defects and the dimension of the nanostructured Si. Si NWs decorated with Si NCs on its surface exhibit strong broadband PL in the visible region at $\mathrm{RT}^{5,6}$. Figure 3 shows the broad visible PL from the samples S1, S1_VA, S1_O2 and S1_N2. The as-grown Si NCs decorated Si NWs (sample S1) show higher PL intensity as compared to the RTA treated samples. Vacuum annealed samples show lowest ( 20 times lower) PL intensity as compared to the as-grown sample. N2 and O2 treated samples show $\sim 6$ times and $\sim 7$ times lower intensity than the as-grown sample. We have studied the effect of NCs morphology on the PL spectra of the NWs ${ }^{5}$. Due to large diameter and indirect nature of the bandgap of the Si NWs, strong visible PL is unlikely to originate from the Si NWs. On the other hand, the dimensions of the self-grown Si NCs are comparable/smaller than the excitonic Bohr diameter in $\mathrm{Si}(\sim 9.8 \mathrm{~nm})$. So the Si NCs are most likely responsible for the visible PL. Often quantum confinement (QC) model is invoked to explain the visible PL from $\mathrm{Si} \mathrm{NCs}^{5,6}$. Low reflectivity also implies higher absorption and excitation of carrier finally leading to enhanced radiative recombination or $\mathrm{PL}^{12}$. 
QC model is the most successful model for explaining size dependent PL spectra of Si nanostructures. From the heterogeneous relations between the diameter (d) of the $\mathrm{Si}$ nanostructure (quasi-1D in Si NW and quasi-0D in Si NC) and corresponding bandgap/ PL peak energy (Eg) expressed as

$$
\mathrm{E}_{\mathrm{g}}(\text { peak })=\mathrm{E}_{\mathrm{g}}(\text { bulk })+\mathrm{Cd}^{-\alpha} \text {, }
$$

where $\mathrm{C}$ and $\alpha$ are constants. Note that the NCs shape is arbitrary with a broad distribution in sizes. Due to the arbitrary shape, we choose the cross-sectional area (A) as the most appropriate parameter to replace the commonly used parameter " $\mathrm{d}$ " resulting in the following equation:

$$
\mathrm{E}_{\mathrm{g}}(\text { peak })=\mathrm{E}_{\mathrm{g}}(\text { bulk })+\mathrm{C}^{\prime} \mathrm{A}^{-\alpha,},
$$

where $C^{\prime}$ and $\alpha^{\prime}$ are new constants. Here $A$ is the area replacing $\pi \mathrm{d}^{2} / 4$ for circular crosssection. To emulate the PL line shape, size distribution of the NCs must be considered. We found ${ }^{5}$ the new constants $\mathrm{C}^{\prime}=3.58 \mathrm{eV} / \mathrm{nm}^{2}$ and $\alpha^{\prime}=0.52$ which are very much consistent to the earlier published reports ${ }^{13}$.

Note that the MACE grown $\mathrm{Si}$ NCs/NWs are covered by a thin layer of $\mathrm{SiO}_{\mathrm{x}}{ }^{5}$, the defects in the $\mathrm{Si} / \mathrm{SiO}_{\mathrm{x}}$ interface can also give rise to visible $\mathrm{PL}$ emission ${ }^{9}$. In order to better understand the origin of the visible PL from the MACE grown Si NWs, we have performed the RTA on the Si NCs decorated Si NWs samples at $800{ }^{\circ} \mathrm{C}$. The annealing environment affects the PL spectra of the Si NCs/NWs and we have fitted the PL spectra of each sample before and after the RTA treatment. The fitting of the PL spectra of these samples give a better idea about the interplay of QC effect in Si NCs and the defects in the interface of $\mathrm{Si} / \mathrm{SiO}_{\mathrm{x}}$ layer. Figures 4 (a-d) show the broad and asymmetric PL spectra for the samples $\mathrm{S} 1$, S1_VA, S1_N2 and S1_O2, respectively, fitted by Gaussian peaks. Fig. 4(a) shows that the PL spectra of the sample S1 consist of two peaks originating from two different groups of Si NCs with different sizes. The size of these Si NCs can be estimated from eqn. (1). Fig. 4(b) 
and 4(c) show that the peaks at $\sim 1.8 \mathrm{eV}$ and $\sim 1.6 \mathrm{eV}$ are common to S1, S1_VA and S1_N2 samples, along with an additional band at $\sim 2.4 \mathrm{eV}$ for all RTA treated samples, i.e., S1_VA, S1_N2 and S1-O2. The green PL emission at $\sim 2.4 \mathrm{eV}$ is possibly due to the neutral oxygen vacancy $\left(\mathrm{V}_{\mathrm{O}}\right)$ related defects present in the $\mathrm{SiO}_{\mathrm{x}}$ layer ${ }^{9}$. Due to the RTA under low vacuum and $\mathrm{N}_{2}$ environment, nonradiative defects may be increased and it results into reduced PL intensity from the Si NCs. In addition, annealing results into increase in oxygen related defects, particularly at the $\mathrm{Si} / \mathrm{SiOx}$ interface and this interface moves inside the $\mathrm{Si} \mathrm{NCs} / \mathrm{NWs}$ core to create newer $\mathrm{SiOx}$ layer, particularly in case of annealing under $\mathrm{O}_{2}$ environment. Thus, the PL band at $2.4 \mathrm{eV}$ is considerably increased in S1_O2 sample. Fig. 4(d) shows the deconvoluted PL spectra of the sample S1_O2 with three Gaussian peaks. Note that PL spectrum of sample S1_O2 is a bit different from the as-grown Si NCs/NWs sample i.e. sample S1. Due to the formation of the new $\mathrm{SiOx}$ layer at the interface of native oxide/ $\mathrm{Si}$ layer during RTA in $\mathrm{O}_{2}$ atmosphere, the $\mathrm{V}_{\mathrm{O}}$ defects are increased and it results into stronger PL peak at $2.4 \mathrm{eV}$. Note that the oxidation of Si NCs/ NWs results in reduction of the size of the original Si NCs/ NWs and this leads to a blue shift in the PL emission peaks origin from the Si NCs. This leads to almost white light emission from the $\mathrm{O} 2$ annealed samples, which is promising for the LED applications. The green emission at $\sim 2.4 \mathrm{eV}$ is due to the neutral $\mathrm{V}_{\mathrm{O}}$ defects in the $\mathrm{SiO}_{x}$ layer and it dominates over the $\mathrm{Si}$ NCs size dependent PL in sample S1_O2. This confirms that the reddish PL emission in the range 1-6-1.8 eV of the as-grown sample is due to the QC effect alone in Si NCs at RT.

In order to understand the effect of laser heating on the PL spectra of the as-grown Si NCs decorated Si NWs, we performed the PL measurements of the sample S1 with different laser excitation power ranging from $5 \mathrm{~mW}$ to $100 \mathrm{~mW}$. Figure 5(a) shows the comparison of the PL spectra of the sample S1 with laser extraction wavelength $405 \mathrm{~nm}$ for different laser excitation power, while 5(b) shows the variation of the PL peak center with increasing the 
laser excitation power (at source). It is clear from Fig. 5(a) that the integrated intensity of the PL spectra increases systematically with increasing the laser power (at source). The PL peak center shows slight red shift with increasing laser power. This is due to the fact that the $\mathrm{Si}$ NCs are heated due to the laser excitation at high power and due to the heating the Si NCs are expanded. Due to the thermal expansion of the Si NCs, size dependent band gap of the Si NCs is reduced according to the eqn. (1), which is consistent with the earlier report by Vershini et $\mathrm{al}^{14}$. Thus, the laser heating effect on PL spectra of the sample S1 confirms that the reddish PL is intrinsic to Si NCs only and can be explained successfully using the QC model.

\subsection{Raman analysis}

Raman scattering is sensitive to the crystal lattice microstructure of $\mathrm{Si}$ via their vibrational properties. Inspection of line shapes of Raman spectra may be the source of useful information concerning the crystalinity, amorphicity, and dimension of nanostructured Si. Therefore, the Raman spectra of the MACE grown Si NWs are of great value for the further understanding of the above parameters. Figure 6(a) shows the Raman spectra in the range 200 - $1100 \mathrm{~cm}^{-1}$ at RT for the samples S1, S1_N2, S1_O2 and S1_VA with that of bulk Si with laser excitation wavelength $633 \mathrm{~nm}$ at laser power $1.8 \mathrm{~mW}$, respectively, while Fig. 6(b) shows the corresponding 1st order Raman spectra (TO phonon mode). The Raman spectra of all the samples were corrected by keeping the first order Raman peak of the bulk $\mathrm{Si}$ at 520.5 $\mathrm{cm}^{-1}\left(\omega_{0}\right)$. Each samples in Fig. 6(a) show the 2TA $\left(290-300 \mathrm{~cm}^{-1}\right)$, TO $\left(510-520.5 \mathrm{~cm}^{-1}\right)$ and 2 TO $\left(950-970 \mathrm{~cm}^{-1}\right)$ Raman phonon modes. All the multi-phonon peaks present in the Si NWs/NCs samples suggest that the MACE grown Si NWs/NCs are indeed highly crystalline in nature. All the spectra of Fig. 6(b) show a red shift of $1^{\text {st }}$ order Raman peak as well as asymmetry towards lower energy as compared to that of the bulk Si. 
The red shift and asymmetric broadening of the $1^{\text {st }}$ order phonon mode of the Raman spectra can be explained in terms of three effects:

(a) Pure QC effect of phonon

(b) QC effect and compressive strain effect

(c) QC effect including compressive strain effect and inhomogeneous laser heating.

In order to avoid the effect of inhomogeneous laser heating due to high laser power, the Raman spectra of the samples is performed with very low excitation power. The Si NWs/NCs are covered with a native oxide layer ${ }^{5}$ and the compressive stress from the oxide matrix could also be responsible for the shifting the $1^{\text {st }}$ order Raman peak to higher frequency and opposing the frequency shift due to phonon confinement ${ }^{15-17}$. From the additional shift of the Raman peaks $\left(\delta \omega=\omega-\omega_{0}\right)$, the lattice stress of the Si can be approximately calculated according to the following equations ${ }^{16}$

$$
\delta \omega=-3 \gamma\left(\mathrm{a}-\mathrm{a}_{0}\right) / \mathrm{a},
$$

where $\mathrm{a}$ is the lattice constant of the strained $\mathrm{Si}, \gamma$ is Gruneisen constant $(\gamma \sim 1.0)$, and $\mathrm{a}_{0}$ is the lattice constant of single-crystalline Si. With the decrease in crystallite size from bulk to nanometer, the special wave function of the optical phonon is confined and the phonon confinement results in the red-shift and asymmetrical broadening of the Raman modes. Assuming that this shift can be fully attributed to QC effect due to the small Si NCs, the $\delta \omega$ can be estimated from the formula ${ }^{18}$

$$
\delta \omega=\mathrm{Ad}^{-2}
$$

where $\mathrm{A}=34.8 \mathrm{~nm}^{2} \mathrm{~cm}^{-1}$. So the actual shift of the TO Raman mode is a competing effect of QC effect of phonon due to Si NCs and compressive strain due to the oxide layer. It is clear from Fig. $6(\mathrm{~b})$ that $\delta \omega$ is large for the as-grown S1 due to the phonon confinement effect, while after RTA $\delta \omega$ is reduced. In case of sample S1_O2, $\delta \omega$ is least. This may be due to the fact that the $\mathrm{Si}$ NWs are covered with a thick layer of $\mathrm{SiO}_{2}$ and Raman spectra originate 
mostly from the $\mathrm{SiO}_{2}$ surface and the $\mathrm{Si}$ inside the $\mathrm{SiO}_{2}$ structure contributes to the $\mathrm{TO}$ mode $1^{\text {st }}$ order Raman spectra and as a result the $\delta \omega$ is almost zero due to the absence of any QC effect.

\section{CONCLUSION}

In conclusion, MACE is carried out to fabricate arrays of Si NWs and these Si NWs are decorated with arbitrary shaped Si NCs that emits reddish PL. Our studies on the effect of RTA treatment on the optical properties of the Si NCs decorated Si NWs confirms that the PL originates from the QC of carrier in Si NCs. Various kinds of point defects are evolved/ reduced depending upon the annealing environment during RTA. The inhomogeneous heating due to high power laser excitation shifts the PL spectra of the Si NCs towards lower energy due to the thermal expansion of the size of the Si NCs. Emission in the yellow-green region was strongly enhanced by RTA treatment in oxygen atmosphere due to the neutral oxygen vacancy defects in the $\mathrm{SiO}_{2}$ layer. This study demonstrate that the RTA treatment is a useful tool for improving optical properties of Si NCs decorated Si NWs and generation of yellow-green LED based on Si nanostructures.

\section{Acknowledgements}

We acknowledge the financial support from CSIR (Grant No. 03(1270)/13/EMR-II) and BRNS (Grant No. 2012/37P/1/BRNS) for carrying out part of this work. Central Instruments Facility (CIF), IIT Guwahati is acknowledged for the HRTEM, FESEM, ellipsometry and micro Raman facilities.

\section{References}

K. Q. Peng and S. T. Lee, Adv. Mater. 23, 198 (2011).

r. Yan, d. Gargas, and p. Yang, Nature Photonics 3, 569 (2009).

K. Peng, J. Jie, W. Zhang, and S. T. Lee, Applied Physics Letters 93, 033105 (2008).

Z. Li, Y. Chen, X. Li, T. I. Kamins, K. Nauka, and R. S. Williams, Nano lett. 4, 245 (2004).

R. Ghosh, P. K. Giri, K. Imakita, and M. Fujii, Nanotechnology 25, 045703 (2014). 
A. S. Kuznetsov, T. Shimizu, S. N. Kuznetsov, A. V. Klekachev, S. Shingubara, J. Vanacken, and V. V. Moshchalkov, Nanotechnology 23, 475709 (2012).

7 G. Ledoux, O. Guillois, D. Porterat, C. Reynaud, F. Huisken, B. Kohn, and V. Paillard, Physical Review B 62, 15942 (2000).

8 T. Suzuki, L. Skuja, K. Kajihara, M. Hirano, T. Kamiya, and H. Hosono, Physical Review Letters 90, 186404 (2003).

9 B. S. Swain, B. P. Swain, S. S. Lee, and N. M. Hwang, The Journal of Physical Chemistry C 116, 22036 (2012).

10 S. Dhara and P. K. Giri, Functional Materials Letters 04, 25 (2011).

11 A. I. Hochbaum, D. Gargas, Y. J. Hwang, and P. Yang, Nano Lett. 9, 3550 (2009).

12 W. Chern, et al., Nano letters 10, 1582 (2010).

13 J. Valenta, B. Bruhn, and J. Linnros, Nano Lett. 11, 3003 (2011).

$14 \quad$ Y. P. Varshni, Physica 34, 149 (1967).

15 C. M. Hessel, J. Wei, D. Reid, H. Fujii, M. C. Downer, and B. A. Korgel, The Journal of Physical Chemistry Letters 3, 1089 (2012).

16 Y. Chen, B. Peng, and B. Wang, The Journal of Physical Chemistry C 111, 5855 (2007).

17 T. Arguirov, T. Mchedlidze, M. Kittler, R. Rölver, B. Berghoff, M. Först, and B. Spangenberg, Applied Physics Letters 89 (2006).

i. Doğan and M. C. M. van de Sanden, Journal of Applied Physics 114 (2013). 
Table I: Details of the growth parameters of the Si NCs decorated Si NWs.

\begin{tabular}{|c|c|c|c|}
\hline \multirow{2}{*}{$\begin{array}{c}\text { Sample } \\
\text { code }\end{array}$} & \multicolumn{3}{|c|}{ Processing parameters } \\
\cline { 2 - 4 } & RTA enviroment & $\begin{array}{c}\text { RTA } \\
\text { temp. }\end{array}$ & $\begin{array}{c}\text { RTA } \\
\text { duration }\end{array}$ \\
\hline S1 & As grown Si NWs & - & - \\
\hline S1_VA & Vacuum & $800^{\circ} \mathrm{C}$ & $60 \mathrm{sec}$ \\
\hline S1_O2 & Oxygen & $800^{\circ} \mathrm{C}$ & $60 \mathrm{sec}$ \\
\hline S1_N2 & Nitrogen & $800^{\circ} \mathrm{C}$ & $60 \mathrm{sec}$ \\
\hline
\end{tabular}
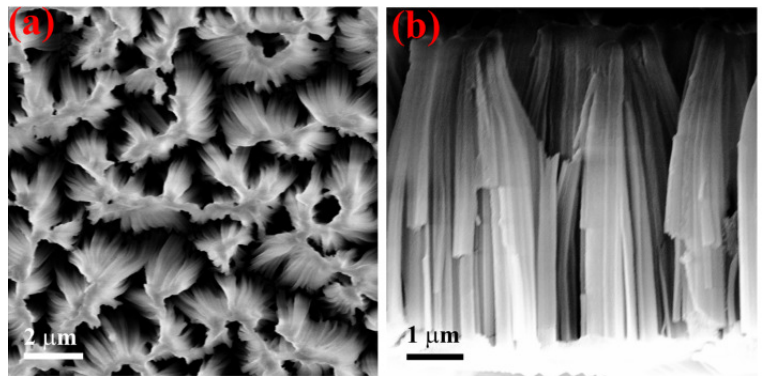

(c)
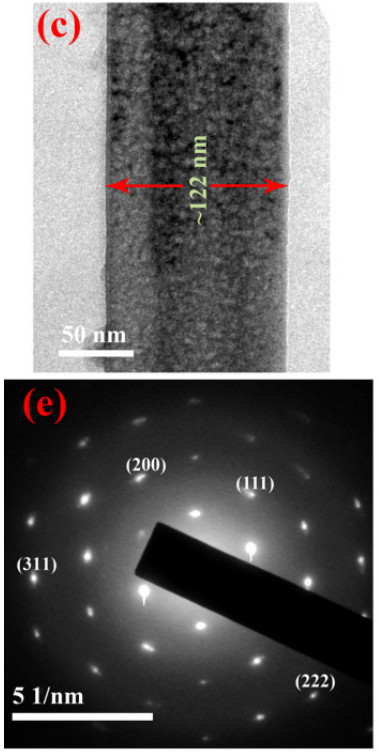

(d)

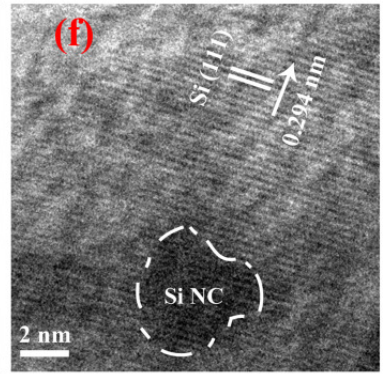

Fig. 1: FESEM (a) top view and (b) cross sectional view of the array of $\mathrm{Si}$ NWs in sample S1. (c) TEM image of a single $\mathrm{Si} \mathrm{NW}$ in sample S1 showing rough surface of the NWs due to sidewall etching and confirming the presence of $\mathrm{Si} \mathrm{NCs}$ on its surface. (d) TEM images of single Si NW for sample S1_O2. A thick layer of $\mathrm{SiO}_{2}(\sim 13 \mathrm{~nm})$ is grown on the surface of the NWs and forms core-shell $\mathrm{Si}_{-} \mathrm{SiO}_{x}$ heterostructure. (e) SAED pattern and (f) HRTEM image of $\mathrm{Si} \mathrm{NW} /$ NCs before RTA. Lattice spacing calculated from the HRTEM image shown in (f) confirms that the (111) Si NWs are compressively strained. 

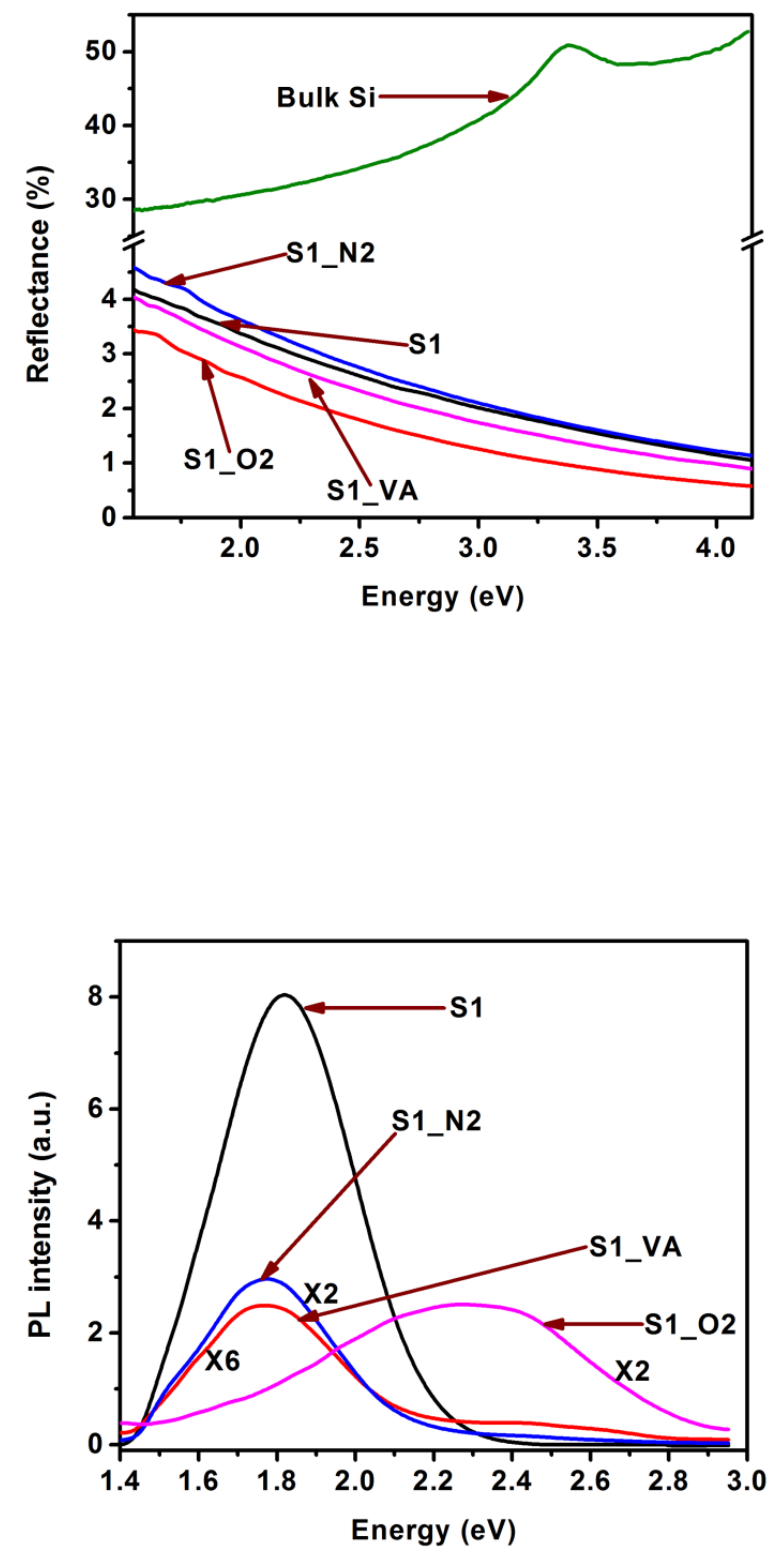

Fig. 2: Comparison of the diffused reflectance spectra for samples S1, S1_VA, S1_O2 and S1_N2 with that of the bulk Si wafer. Si NCs/NWs before and after RTA treatment in different annealing atmosphere have exceptionally low reflectance in a wide visible-NIR range.
Fig. 3: Comparison of the PL spectra recorded with $\lambda_{\text {ex }}=405 \mathrm{~nm}$ for samples S1, S1_VA, S1_O2 and S1_N2. The PL spectra of the samples S1_VA, S1_O2 and S1_N2 are scaled up by appropriate factors to enable comparison. 

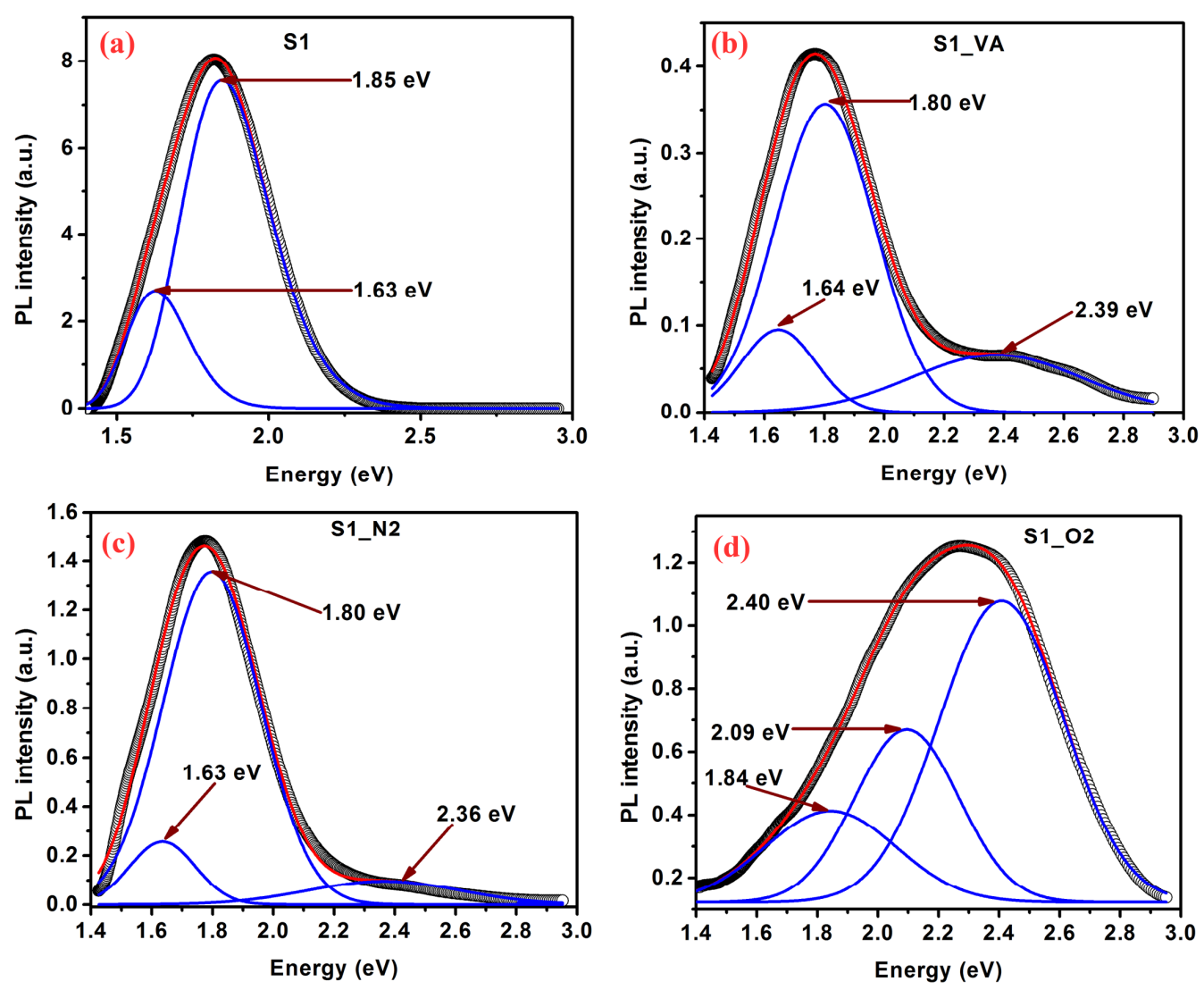

Fig. 4: The PL spectra along with Gaussian line shape fitting for samples (a) S1, (b) S1_VA, (c) S1_N2 and (d) S1_O2, acquired with $\lambda_{\mathrm{ex}}=405 \mathrm{~nm}$. The experimental data is shown with symbols and the fitted curves are shown with solid lines. The peak centers are denoted in each case. 


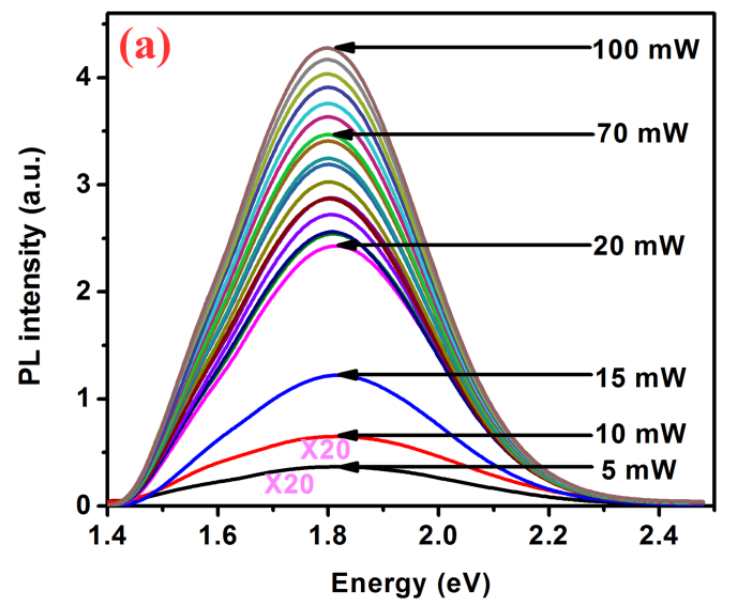

Fig. 5: (a) Comparison of the PL spectra of the sample $\mathrm{S} 1$ with $\lambda_{\mathrm{ex}}=$ $405 \mathrm{~nm}$ for different laser excitation power in the range $5-100 \mathrm{~mW}$, in steps of $5 \mathrm{~mW}$. The PL spectra with laser excitation power 5 and $10 \mathrm{~mW}$ are scaled up by 20 to enable comparison. (b) Variation of the PL peak center with the laser excitation power.

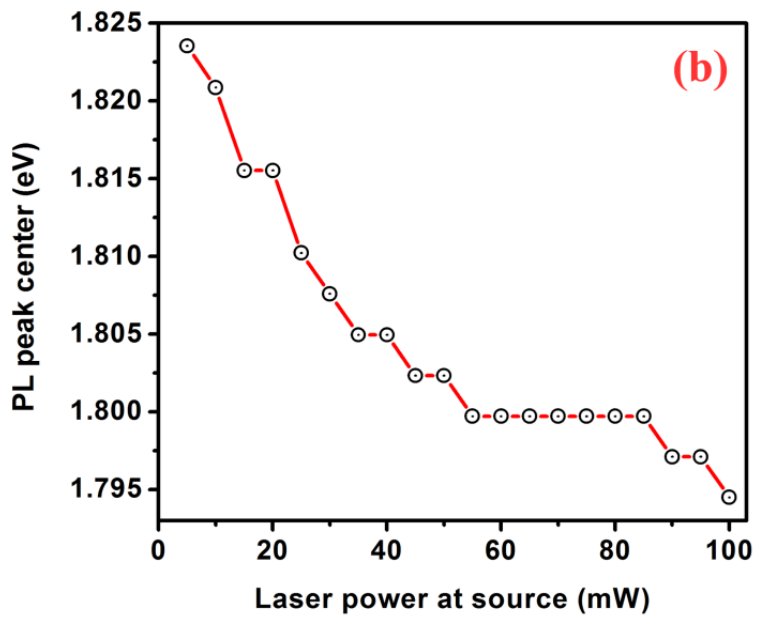




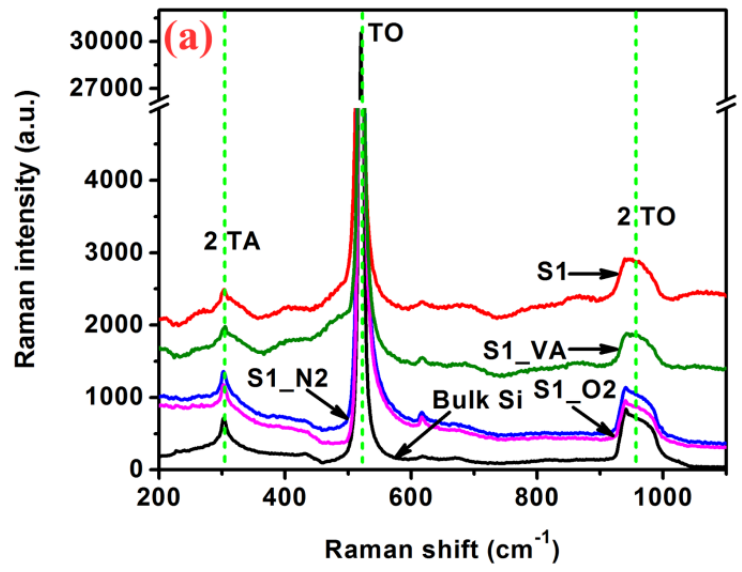

Fig. 6: (a) Comparison of the Raman spectra of the samples S1, S1_VA, S1_O2 and S1_N2. (b) Comparison of $1^{\text {st }}$ order Raman spectra (TO phonon mode) for the above samples. For comparison, the Raman spectrum of the bulk $\mathrm{Si}$ is also shown. The multiphonon peaks are shown with dotted vertical lines.

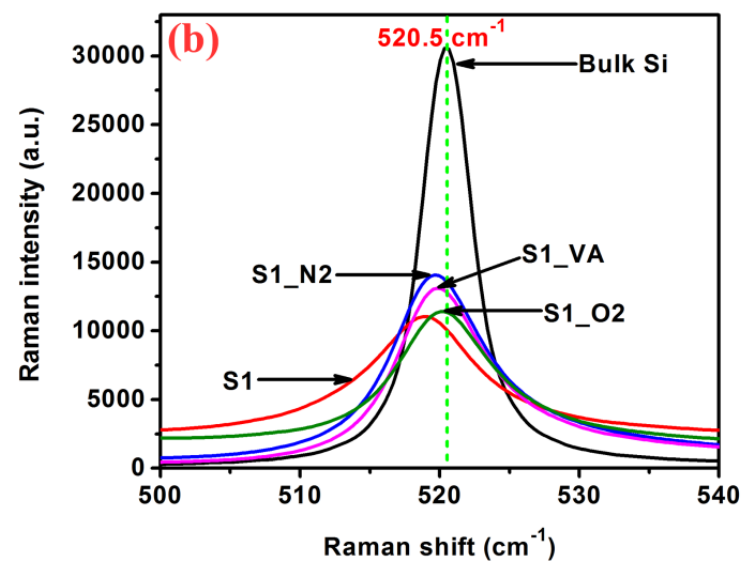

